\title{
Existence and convergence theorems for the new system of generalized mixed variational inequalities in Banach spaces
}

\author{
Nawitcha Onjai-uea ${ }^{1,2}$ and Poom Kumam ${ }^{1,2^{*}}$
}

* Correspondence: poom.
kum@kmutt.ac.th
'Department of Mathematics,
Faculty of Science, King Mongkut's
University of Technology Thonburi
(KMUTT), Bangkok 10140, Thailand
Full list of author information is
available at the end of the article

available at the end of the article

\begin{abstract}
In this article, we introduce and consider the three-step iterative algorithms for solving a new system of generalized mixed variational inequalities involving different three multi-valued operators. In this study, we use a generalized f-projection method for finding the solutions of generalized system of mixed variational inequalities in Banach spaces. Our result in this article improves and generalizes some known corresponding results in the literature.

2000 Mathematics Subject Classification: 47H10; 47H19; $49 J 40$.
\end{abstract}

Keywords: a new generalized system mixed variational inequality problem, relaxed cocoercive mappings, fixed point problem, generalized $f$-projection

\section{Introduction}

Let $B$ be a real Banach space which dual space $B^{*}$ and $C$ be a nonempty closed convex subset of $B$. Let $\langle\cdot, \cdot\rangle$ be the dual pair between $B$ and $B^{*}, J$ denotes the normalized duality mapping and $\mathbb{R}$ be the field of real numbers. Let $T_{1}, T_{2}, T_{3}: C \rightarrow 2^{B^{*}}$ be nonlinear operators. Let $f_{1}, f_{2}, f_{3}: B \rightarrow(-\infty,+\infty]$ be three mappings. We consider the following problem:

Find $x^{*}, y^{*}, z^{*} \in C \subset B$ such that there exist $u^{*} \in T_{1}\left(y^{*}\right), v^{*} \in T_{2}\left(z^{*}\right)$, and $w^{*} \in T_{3}\left(x^{*}\right)$ satisfying

$$
(\star)\left\{\begin{array}{l}
\left\langle u^{*}+J x^{*}-J y^{*}, x-x^{*}\right\rangle+f_{1}(x)-f_{1}\left(x^{*}\right) \geq 0, \quad \forall x \in C, \\
\left.v^{*}+J y^{*}-J z^{*}, x-y^{*}\right\rangle+f_{2}(x)-f_{2}\left(y^{*}\right) \geq 0, \quad \forall x \in C, \\
\left\langle w^{*}+J z^{*}-J x^{*}, x-z^{*}\right\rangle+f_{3}(x)-f_{3}\left(z^{*}\right) \geq 0, \forall x \in C .
\end{array}\right.
$$

The problem $(\star)$ is called the system of generalized mixed variational inequality problems, the solution of $(\star)$ is denoted by (SGMVIP).

Some special cases of the problem $(\star)$ :

(I) If $f_{1}(x)=f_{2}(x)=f_{3}(x)=0, \forall x \in C$, then problem $(\star)$ is equivalent to find $x^{*}, y^{*}$, $z^{*} \in C$ such that

$$
\left\{\begin{array}{l}
\left\langle u^{*}+J x^{*}-J y^{*}, x-x^{*}\right\rangle \geq 0, \quad \forall x \in C, \\
\left\langle v^{*}+J y^{*}-J z^{*}, x-y^{*}\right\rangle \geq 0, \quad \forall x \in C, \\
\left\langle w^{*}+J z^{*}-J x^{*}, x-z^{*}\right\rangle \geq 0, \forall x \in C
\end{array}\right.
$$

\section{Springer}

(c) 2012 Onjai-uea and Kumam; licensee Springer. This is an Open Access article distributed under the terms of the Creative Commons Attribution License (http://creativecommons.org/licenses/by/2.0), which permits unrestricted use, distribution, and reproduction in any medium, provided the original work is properly cited. 
The problem (1.1) is called the system of generalized variational inequality problems, the solution of (1.1) is denoted by (SGVIP).

(II) If $x^{*}=z^{*}, T_{3}(y)=0$, and $f_{3}(x)=0, \forall x, y \in C$, then problem ( $\star$ ) is equivalent to the two step of the system of generalized mixed variational inequality problems: Find $x^{*}, y^{*} \in C \subset B$ such that there exist $u^{*} \in T_{1}\left(y^{*}\right)$ and $v^{*} \in T_{2}\left(x^{*}\right)$ satisfying

$$
\left\{\begin{array}{l}
\left\langle u^{*}+J x^{*}-J y^{*}, x-x^{*}\right\rangle+f_{1}(x)-f_{1}\left(x^{*}\right) \geq 0, \forall x \in C, \\
\left\langle v^{*}+J y^{*}-J x^{*}, x-y^{*}\right\rangle+f_{2}(x)-f_{2}\left(y^{*}\right) \geq 0, \forall x \in C
\end{array}\right.
$$

which was studied by Zhang and Deng [1].

(III) If $T=T_{1}=T_{2}=T_{3}, f_{2}(x)=f_{3}(x)=0, \forall x \in C$ and $x^{*}=y^{*}=z^{*}$, then problem ( $\star$ ) is equivalent to the generalized variational inequality problem associated with $C, T$, and $f$ denoted by $\operatorname{GVI}(C, T, f)$ : find $x^{*} \in C$ such that there exist $u^{*} \in T\left(x^{*}\right)$ satisfying

$$
\left\langle u^{*}, x-x^{*}\right\rangle+f_{1}(x)-f_{1}\left(x^{*}\right) \geq 0, \quad \forall x \in C
$$

which was studied by Fan et al. [2].

If $f_{1}(x)=0, \forall x \in C$ and $T$ is single-valued, then problem (1.3) reduces to the classical variational inequality problem, which consists in finding $x \in C$ such that

$$
\left\langle T x, x-x^{*}\right\rangle \geq 0, \quad \forall x \in C
$$

which is known as the classical variational inequality introduced and studied by Stampacchia [3] in 1964. For the recent applications, numerical methods and formulations, (see for example [3-10]) and the references therein. The variational inequalities are equivalent to the fixed point problems. In particular, the solution of the variational inequalities can be computed using the iterative projection method. Alber [11] presented some applications of the generalized projections to approximately solving variational inequalities and von Neumann intersection problem in Banach spaces. In 2005, Li [12] extended the generalized projection operator from uniformly convex and uniformly smooth Banach spaces to reflexive Banach spaces and studied some properties of the generalized projection operator with applications to solving the variational inequality in Banach spaces.

In 2007, Wu and Huang [13], they proved some properties of the generalized $f$-projection operator and proposed iterative method of approximating solutions for a class of generalized variational inequalities in Banach spaces. In 2009, Fan et al. [2] presented some basic results for the generalized $f$-projection operator and discussed the existence of solutions and approximation of the solutions for generalized variational inequalities in noncompact subsets of Banach spaces. In 2010, Petrot [8] used the resolvent operator technique to find the common solutions for a generalized system of relaxed cocoercive mixed variational inequality problems and fixed point problems for Lipschitz mappings in Hilbert spaces.

In 2011, Zhang and Deng [1] introduced and considered the system of mixed variational inequalities in Banach spaces. Using the generalized $f$-projection operator technique, they introduced two-step iterative methods for solving the system of mixed variational inequalities and proved the convergence of the proposed iterative methods under suitable conditions in Banach spaces.

Noor [4] suggested and analyzed several three-step iterative methods, which are also known as Noor iterations, for solving variational inequalities. It has been shown that 
three-step iterative methods are more efficient than two-step and one-step iterative methods. In addition, it is known that the convergence analysis of three-step can be proved under much weaker conditions.

Motivated and inspired by the recent research studies in this fascinating area, the purpose of this article is to introduce and analyze three-step iterative algorithm for finding a new system of generalized mixed variational inequality problems with three difference multi-valued operators in Banach spaces. Using the generalized $f$-projection method. The results presented in this article extend and improve the results of Zhang and Deng [1] and Fan et al. [2] and some authors.

\section{Preliminaries}

A Banach space $B$ is said to be strictly convex if $\left\|\frac{x+y}{2}\right\|<1$ for all $x, y \in B$ with $\|x\|=$ $\|y\|=1$ and $x \neq y$. Let $U=\{x \in B:\|x\|=1\}$ be the unit sphere of $B$. then a Banach space $B$ is said to be smooth if the $\lim _{t \rightarrow 0} \frac{\|x+t y\|-\|x\|}{t}$ exists for each $x, y \in U$. It is also said to be uniformly smooth if the limit exists uniformly in $x, y \in U$. Let $B$ be a Banach space. The modulus of smoothness of $B$ is the function $\rho_{B}:[0, \infty) \rightarrow[0, \infty)$ defined by $\rho_{B}(t)=\sup \left\{\frac{\|x+y\|+\|x-y\|}{2}-1:\|x\|=1,\|y\| \leq t\right\}$. The modulus of convexity of $B$ is the function $\eta_{B}:[0,2] \rightarrow[0,1]$ defined by $\eta_{B}(\varepsilon)=\inf \left\{1-\left\|\frac{x+y}{2}\right\|: x, y \in B,\|x\|=\|y\|=1,\|x-y\| \geq \varepsilon\right\}$. The normalized duality mapping $J: B \rightarrow 2^{E^{*}}$ is defined by $J(x)=\left\{x^{*} \in B^{*}:\left\langle x, x^{*}\right\rangle=\|x\|^{2},\left\|x^{*}\right\|=\|x\|\right\}$. If $B$ is a Hilbert space, then $J=I$, where $I$ is the identity mapping.

If $B$ is a reflexive smooth and strictly convex Banach space and $F^{*}: B^{*} \rightarrow 2^{B}$ is the normalized duality mapping on $B^{*}$, then $J^{-1}=J^{*}, J J^{*}=I_{B^{*}}$ and $J^{*} J=I_{B}$, where $I_{B}$ and $I_{B}^{*}$ are the identity mappings on $B$ and $B^{*}$. If $B$ is a uniformly smooth and uniformly convex Banach space, then $J$ is uniformly norm-to-norm continuous on bounded subsets of $B$ and $F^{*}$ is also uniformly norm-to-norm continuous on bounded subsets of $B^{*}$.

Let $B$ and $F$ be Banach spaces, $T: D(T) \subset B \rightarrow F$, the operator $T$ is said to be compact if it is continuous and maps the bounded subsets of $D(T)$ onto the relatively compact subsets of $F$; the operator $T$ is said to be weak to norm continuous if it is continuous from the weak topology of $B$ to the strong topology of $F$.

We also need the following lemmas for the proof of our main results.

Lemma 2.1. [14]. Let $q>1$ and $r>0$ be two fixed real numbers. Let $B$ be a uniformly convex Banach space if and only if there exists a continuous strictly increasing and convex function $g:[0,+\infty) \rightarrow[0,+\infty), g(0)=0$ such that

$$
\|\lambda x+(1-\lambda) y\|^{q} \leq \lambda\|x\|^{q}+(1-\lambda)\|y\|^{q}-\varsigma_{q}(\lambda) g(\|x-\gamma\|)
$$

for all $x, y \in B_{r}=\{x \in B:\|x\| \leq r\}$ and $\lambda \in[0,1]$, where $\varsigma_{q}(\lambda)=\lambda(1-\lambda)^{q}+\lambda^{q}(1-\lambda)$. For case $q=2$, we have

$$
\|\lambda x+(1-\lambda) y\|^{2} \leq \lambda\|x\|^{2}+(1-\lambda)\|y\|^{2}-\lambda(1-\lambda) g(\|x-y\|) .
$$

Lemma 2.2. [15]. Let B be a uniformly convex and uniformly smooth Banach space. We have

$$
\|\phi+\Phi\|^{2} \leq\|\phi\|^{2}+2\left\langle\Phi, J^{*}(\phi+\Phi)\right\rangle, \quad \forall \phi, \Phi \in E^{*} .
$$


Next, we recall the concept of the generalized $f$-projection operator. Let $G: B^{*} \times C \rightarrow$ $\mathbb{R} \cup\{+\infty\}$ be a functional defined as follows:

$$
G(\xi, x)=\|\xi\|^{2}-2\langle\xi, x\rangle+\|x\|^{2}+2 \rho f(x),
$$

where $\xi \in B^{*}, \rho$ is positive number and $f: C \rightarrow \mathbb{R} \cup\{+\infty\}$ is proper, convex, and lower semi-continuous. From definitions of $G$ and $f$, it is easy to see the following properties:

(i) $(\|\xi\|-\|x\|)^{2}+2 \rho f(x) \leq G(\xi, x) \leq(\|\xi\|+\|x\|)^{2}+2 \rho f(x)$;

(ii) $G(\xi, x)$ is convex and continuous with respect to $x$, when $\xi$ is fixed;

(iii) $G(\xi, x)$ is convex and lower semicontinuous with respect to $\xi$, when $x$ is fixed.

Definition 2.3. Let $B$ be a real Banach space with its dual $B^{*}$. Let $C$ be a nonempty closed convex subset of $B$. We say that $\pi_{C}^{f}: B^{*} \rightarrow 2^{C}$ is a generalized f-projection operator if

$$
\pi_{C}^{F} \xi=\left\{u \in C: G(\xi, u)=\inf _{\gamma \in C} G(\xi, y)\right\}, \quad \forall \xi \in B^{*} .
$$

In this article, we fixed $\rho=1$, we have

$$
G(\xi, x)=\|\xi\|^{2}-2\langle\xi, x\rangle+\|x\|^{2}+2 f(x) .
$$

For the generalized $f$-projection operator, Wu and Hung [13] proved the following basic properties.

Lemma 2.4. [16]. Let $B$ be a reflexive Banach space with its dual $B^{*}$ and let $C$ be a nonempty closed convex subset of B. The following statement holds:

(i) $\pi_{C}^{f}$ is nonempty closed convex subset of $C$ for all $\xi \in B^{*}$;

(ii) if $B$ is smooth, then for all $\xi \in E^{*}, x \in \pi_{C}^{f} \xi i f$ and only if

$$
\langle\xi-J x, x-y\rangle+\rho f(y)-\rho f(x) \geq 0, \quad \forall y \in C ;
$$

(iii) if $B$ is smooth, then for any $\xi \in B^{*}, \pi_{C}^{f} \xi=(J+\rho \partial f)^{-1} \xi$, where $\partial f$ is the subdifferential of the proper convex and lower semi-continuous functional $f$.

Lemma 2.5. [16]. If $f(x) \geq 0$ for all $x \in C$, then for any $\rho>0$, we have

$$
G(J x, y) \leq G(\xi, y)+2 \rho f(y), \quad \forall \xi \in B^{*}, y \in C, x \in \pi_{C}^{f} \xi .
$$

Lemma 2.6. [2]. Let $B$ be a reflexive strictly convex Banach space with its dual $B^{*}$ and let $C$ be a nonempty closed convex subset of $B$. If $f: C \rightarrow \mathbb{R} \cup\{+\infty\}$ is proper, convex, and lower semi-continuous, then

(i) $\pi_{C}^{f}: B^{*} \rightarrow$ Cis single valued and norm to weak continuous;

(ii) if $B$ has the property (h), that is, for any sequence $\left\{x_{n}\right\} \subset B, x_{n}-x \in E$ and $\|$ $x_{n}\|\rightarrow\| x \|$, implies $x_{n} \rightarrow x$, then $\pi_{C}^{f}: B^{*} \rightarrow$ Cis continuous. 
Defined the functional $G_{2}: B \times C \rightarrow \mathbb{R} \cup\{+\infty\}$ by

$$
G_{2}(x, y)=G(J x, y), \forall x \in B, y \in C .
$$

\section{Algorithms}

First, we establish a useful Lemma for solving the new system of generalized mixed variational inequalities is equivalent to find a fixed point of generalized $f$-projection operator. For this purpose, we recall the following result.

Lemma 3.1. Let $C$ be nonempty subset of a reflexive, strictly convex and smooth Banach space $B$. If $f_{1}, f_{2}, f_{3}: C \rightarrow(-\infty,+\infty]$ are proper, convex, and lower semi-continuous, then $\left(x^{*}, y^{*}, z^{*}\right)$ is a solution of problem $(\star)$ is equivalent to find $x^{*}, y^{*}$, and $z^{*}$ such that $u^{*} \in T_{1}\left(y^{*}\right), v^{*} \in T_{2}\left(z^{*}\right), w^{*} \in T_{3}\left(x^{*}\right)$ and

$$
\left\{\begin{array}{l}
x^{*}=\pi_{C}^{f_{1}}\left(J y^{*}-u^{*}\right) \\
y^{*}=\pi_{C}^{f_{2}}\left(J z^{*}-v^{*}\right) \\
z^{*}=\pi_{C}^{f_{3}}\left(J x^{*}-w^{*}\right) .
\end{array}\right.
$$

Proof. Since $B$ is a reflexive strictly convex and smooth Banach space, we know that $J$ is single-valued and $\pi_{C}^{f_{i}}(i=1,2,3)$ is well defined and single valued. In fact, $\left(x^{*}, y^{*}, z^{*}\right)$ is a solution of problem $(\star)$ if and only if

$$
\begin{array}{ll}
\left\langle u^{*}+J x^{*}-J y^{*}, x-x^{*}\right\rangle+f_{1}(x)-f_{1}\left(x^{*}\right) \geq 0, & \forall x \in C, \\
\left\langle v^{*}+J y^{*}-J z^{*}, x-y^{*}\right\rangle+f_{2}(x)-f_{2}\left(y^{*}\right) \geq 0, & \forall x \in C, \\
\left\langle w^{*}+J z^{*}-J x^{*}, x-z^{*}\right\rangle+f_{3}(x)-f_{3}\left(z^{*}\right) \geq 0, & \forall x \in C
\end{array}
$$

if and only if for all $x \in C$,

$$
\begin{aligned}
& \left\langle\left(J y^{*}-u^{*}\right)-J x^{*}, x-x^{*}\right\rangle+f_{1}(x)-f_{1}\left(x^{*}\right) \geq 0, \quad \forall x \in C, \\
& \left\langle\left(J z^{*}-v^{*}\right)-J y^{*}, y-x\right\rangle+f_{2}(x)-f_{2}\left(y^{*}\right) \geq 0, \quad \forall x \in C, \\
& \left\langle\left(J x^{*}-w\right)-J z^{*}, z^{*}-x\right\rangle+f_{3}(x)-f_{3}\left(z^{*}\right) \geq 0, \quad \forall x \in C .
\end{aligned}
$$

By Lemma 2.4 (ii), if and only if

$$
\left\{\begin{array}{l}
x *=\pi_{C}^{f_{1}}(J y *-u *), \\
y *=\pi_{C}^{f_{2}}(J z *-v *), \\
z *=\pi_{C}^{f_{3}}(J x *-w *) .
\end{array}\right.
$$

This complete the proof.

Algorithm 3.2. For arbitrarily chosen initial points $x_{0}, y_{0}, z_{0} \in C$; compute the sequences $\left\{x_{n}\right\},\left\{y_{n}\right\},\left\{z_{n}\right\}$ such that

$$
\left\{\begin{array}{l}
w_{n} \in T_{3} x_{n}, u_{n} \in T_{1} y_{n}, v_{n} \in T_{2} z_{n}, \\
z_{n}=\left(1-\gamma_{n}\right) x_{n}+\gamma_{n} \pi_{C}^{f_{3}}\left(J x_{n}-w_{n}\right), \\
y_{n}=\left(1-\beta_{n}\right) x_{n}+\beta_{n} \pi_{C}^{f_{2}}\left(J z_{n}-v_{n}\right), \\
x_{n+1}=\left(1-\alpha_{n}\right) x_{n}+\alpha_{n} \pi_{C}^{f_{1}}\left(J y_{n}-u_{n}\right),
\end{array}\right.
$$

where $\left\{\alpha_{n}\right\},\left\{\beta_{n}\right\},\left\{\gamma_{n}\right\} \subset[0,1], \forall n \geq 0$.

Algorithm 3.3. For arbitrarily chosen initial points $x_{0}, y_{0}, z_{0} \in C$; compute the sequences $\left\{x_{n}\right\},\left\{y_{n}\right\},\left\{z_{n}\right\}$ such that 


$$
\left\{\begin{array}{l}
w_{n} \in T_{3} x_{n}, u_{n} \in T_{1} y_{n}, v_{n} \in T_{2} z_{n}, \\
z_{n}=\pi_{C}^{f_{3}}\left(J x_{n}-w_{n}\right), \\
y_{n}=\pi_{C}^{f_{2}}\left(J z_{n}-v_{n}\right), \\
x_{n+1}=\left(1-\alpha_{n}\right) x_{n}+\alpha_{n} \pi_{C}^{f_{1}}\left(J y_{n}-u_{n}\right),
\end{array}\right.
$$

where $\left\{\alpha_{n}\right\} \subset[0,1], \forall n \geq 0$.

If $f_{1}(x)=f_{2}(x)=f_{3}(x)=0, \forall x \in C$, then Algorithm 3.2 reduces to the following iterative method for solving (SGVIP) problem (1.1).

Algorithm 3.4. For arbitrarily chosen initial points $x_{0}, y_{0}, z_{0} \in C$; compute the sequences $\left\{x_{n}\right\},\left\{y_{n}\right\},\left\{z_{n}\right\}$ such that

$$
\left\{\begin{array}{l}
w_{n} \in T_{3} x_{n}, u_{n} \in T_{1} y_{n}, v_{n} \in T_{2} z_{n} \\
z_{n}=\left(1-\gamma_{n}\right) x_{n}+\gamma_{n} \pi_{C}\left(J x_{n}-w_{n}\right), \\
y_{n}=\left(1-\beta_{n}\right) x_{n}+\beta_{n} \pi_{C}\left(J z_{n}-v_{n}\right) \\
x_{n+1}=\left(1-\alpha_{n}\right) x_{n}+\alpha_{n} \pi_{C}\left(J y_{n}-u_{n}\right)
\end{array}\right.
$$

where $\left\{\alpha_{n}\right\},\left\{\beta_{n}\right\},\left\{\gamma_{n}\right\} \subset[0,1], \forall n \geq 0$.

If $\gamma_{n}=0, f_{3}(x)=0$ and $T_{3}(y)=0, \forall x, y \in C$, then Algorithm 3.2 reduces to the following iterative method for solving problem (1.2).

Algorithm 3.5. For arbitrarily chosen initial points $x_{0}, y_{0}, \in C$; compute the sequences $\left\{x_{n}\right\},\left\{y_{n}\right\}$ such that

$$
\left\{\begin{array}{l}
u_{n} \in T_{1} y_{n}, v_{n} \in T_{2} x_{n} \\
y_{n}=\left(1-\beta_{n}\right) x_{n}+\beta_{n} \pi_{C}^{f_{2}}\left(J x_{n}-v_{n}\right) \\
x_{n+1}=\left(1-\alpha_{n}\right) x_{n}+\alpha_{n} \pi_{C}^{f_{1}}\left(J y_{n}-u_{n}\right)
\end{array}\right.
$$

where $\left\{\alpha_{n}\right\},\left\{\beta_{n}\right\} \subset[0,1], \forall n \geq 0$.

If $\beta_{n}=\gamma_{n}=0, T=T_{1}=T_{2}=T_{3}, f_{2}(x)=f_{3}(x)=0, \forall x \in C$, then Algorithm 3.2 reduces to the following iterative method for solving problem (1.3).

Algorithm 3.6. For arbitrarily chosen initial points $x_{0} \in C$; compute the sequence $\left\{x_{n}\right\}$ such that

$$
\left\{\begin{array}{l}
u_{n} \in T x_{n} \\
x_{n+1}=\left(1-\alpha_{n}\right) x_{n}+\alpha_{n} \pi_{C}^{f_{1}}\left(J x_{n}-u_{n}\right)
\end{array}\right.
$$

where $\left\{\alpha_{n}\right\} \subset[0,1], \forall n \geq 0$.

\section{Existence and Convergence Theorems}

Now, we state and prove the main results of this study.

Theorem 4.1. Let $C$ be a nonempty, closed convex subset of a uniformly convex and uniformly smooth Banach space $B$ with dual space $B^{*}$ and $0 \in C$. If the upper semicontinuous set-valued mappings $T_{1}, T_{2}, T_{3}: C \rightarrow 2^{B^{*}}$ with closed values and the proper convex lower semi-continuous mapping $f_{1}, f_{2}, f_{3}: C \rightarrow \mathbb{R} \cup\{+\infty\}$ satisfy the following conditions:

(i) $f_{k}(x) \geq 0$ for all $x \in C$ and $f_{k}(0)=0$ for $k=1,2,3$;

(ii) for any $x \in C$ and any $z_{k} \in T_{k}(x),\left\langle z_{k}, J^{*}\left(J x-z_{k}\right)\right\rangle \geq 0$ for $k=1,2,3$;

(iii) the set-valued mappings $J-T_{k}$ are compact for $k=1,2,3$;

(iv) $0<a \leq \alpha_{n} \leq b<1,0<c \leq \beta_{n} \leq d<1$ and $0<e \leq \gamma_{n} \leq h<1, \exists a, b, c, d, e, h \in$ $(0,1)$. 
Then problem $(\star)$ has a solution $\left(x^{*}, y^{*}, z^{*}\right)$ and the sequences $\left\{x_{n}\right\},\left\{y_{n}\right\}$, and $\left\{z_{n}\right\}$ defined by Algorithm 3.2 have convergent subsequences $\left\{x_{n_{i}}\right\},\left\{y_{n_{i}}\right\}$, and $\left\{z_{n_{i}}\right\}$ such that $x_{n_{i}} \rightarrow x^{*}, y_{n_{i}} \rightarrow y^{*}$, and $z_{n_{i}} \rightarrow z^{*}$ as $i \rightarrow \infty$, respectively.

Proof. Since $B$ is a uniformly convex and uniformly smooth Banach space, we know that $J$ is a bijection from $B$ onto $B^{*}$ and uniformly continuous on any bounded subsets of $B$. Hence $\pi_{C}^{f_{k}}$ is well defined and single-valued and the sequences $\left\{x_{n}\right\},\left\{y_{n}\right\}$ and $\left\{z_{n}\right\}$ are well defined.

Let $G_{2}(x, y)=G(J x, y)$. Then, for any $x \in C$,

$$
G_{2}(x, 0)=G(J x, 0)=\|J x\|^{2}-2\langle J x, 0\rangle+2 f_{k}(0)=\|x\|^{2}, \quad k=1,2,3 .
$$

From Lemma 2.5 and the above equation, we have

$$
\begin{aligned}
\left\|\pi_{C}^{f_{1}}\left(J y_{n}-u_{n}\right)\right\|^{2} & =G_{2}\left(\pi_{C}^{f_{1}}\left(J y_{n}-u_{n}\right), 0\right) \\
& =G\left(J\left(\pi_{C}^{f_{1}}\left(J y_{n}-u_{n}\right)\right), 0\right) \\
& \leq G\left(J y_{n}-u_{n}, 0\right)=\left\|J y_{n}-u_{n}\right\|^{2} .
\end{aligned}
$$

Similarly proof, we also have

$$
\left\|\pi_{C}^{f_{2}}\left(J z_{n}-v_{n}\right)\right\|^{2} \leq\left\|J z_{n}-v_{n}\right\|^{2}
$$

and

$$
\left\|\pi_{C}^{f_{3}}\left(J x_{n}-w_{n}\right)\right\|^{2} \leq\left\|J x_{n}-w_{n}\right\|^{2} .
$$

By Lemma 2.2 and condition (ii), we obtain

$$
\begin{aligned}
& \left\|J y_{n}-u_{n}\right\|^{2} \leq\left\|J y_{n}\right\|^{2}-2\left\langle u_{n}, J^{*}\left(J y_{n}-u_{n}\right)\right\rangle \leq\left\|y_{n}\right\|^{2}, \\
& \left\|J z_{n}-v_{n}\right\|^{2} \leq\left\|J z_{n}\right\|^{2}-2\left\langle v_{n}, J^{*}\left(J z_{n}-v_{n}\right)\right\rangle \leq\left\|z_{n}\right\|^{2}, \\
& \left\|J x_{n}-w_{n}\right\|^{2} \leq\left\|J x_{n}\right\|^{2}-2\left\langle w_{n}, J^{*}\left(J x_{n}-w_{n}\right)\right\rangle \leq\left\|x_{n}\right\|^{2} .
\end{aligned}
$$

It follows that

$$
\begin{aligned}
\left\|z_{n}\right\| & =\left\|\left(1-\gamma_{n}\right) x_{n}+\gamma_{n} \pi_{C}^{f_{3}}\left(J x_{n}-w_{n}\right)\right\| \\
& \leq\left(1-\gamma_{n}\right)\left\|x_{n}\right\|+\gamma_{n}\left\|\pi_{C}^{f_{3}}\left(J x_{n}-w_{n}\right)\right\| \\
& \leq\left(1-\gamma_{n}\right)\left\|x_{n}\right\|+\gamma_{n}\left\|x_{n}\right\|=\left\|x_{n}\right\|, \\
\left\|y_{n}\right\| & =\left\|\left(1-\beta_{n}\right) x_{n}+\beta_{n} \pi_{C}^{f_{2}}\left(J z_{n}-v_{n}\right)\right\| \\
& \leq\left(1-\beta_{n}\right)\left\|x_{n}\right\|+\beta_{n}\left\|\pi_{C}^{f_{2}}\left(J z_{n}-v_{n}\right)\right\| \\
& \leq\left(1-\beta_{n}\right)\left\|x_{n}\right\|+\beta_{n}\left\|z_{n}\right\| \\
& \leq\left(1-\beta_{n}\right)\left\|x_{n}\right\|+\beta_{n}\left\|x_{n}\right\|=\left\|x_{n}\right\|
\end{aligned}
$$

and

$$
\begin{aligned}
\left\|x_{n+1}\right\| & =\left\|\left(1-\alpha_{n}\right) x_{n}+\alpha_{n} \pi_{C}^{f_{1}}\left(J y_{n}-u_{n}\right)\right\| \\
& \leq\left(1-\alpha_{n}\right)\left\|x_{n}\right\|+\alpha_{n}\left\|\pi_{C}^{f_{1}}\left(J y_{n}-u_{n}\right)\right\| \\
& \leq\left(1-\alpha_{n}\right)\left\|x_{n}\right\|+\alpha_{n}\left\|y_{n}\right\| \\
& \leq\left(1-\alpha_{n}\right)\left\|x_{n}\right\|+\alpha_{n}\left\|x_{n}\right\|=\left\|x_{n}\right\| .
\end{aligned}
$$


Hence, the sequences $\left\{x_{n}\right\},\left\{y_{n}\right\},\left\{z_{n}\right\},\left\{\pi_{C}^{f_{1}}\left(J y_{n}-u_{n}\right)\right\},\left\{\pi_{C}^{f_{2}}\left(J z_{n}-v_{n}\right)\right\}$, and $\left\{\pi_{C}^{f_{3}}\left(J x_{n}-w_{n}\right)\right\}$ are bounded. So, we take a positive number $r_{1}, r_{2}, r_{3}, r_{4}, r_{5}, r_{6}$ such that $\left\|x_{n}\right\| \leq r_{1},\left\|y_{n}\right\| \leq r_{2},\left\|z_{n}\right\| \leq r_{3},\left\|\pi_{C}^{f_{1}}\left(J y_{n}-u_{n}\right)\right\| \leq r_{4},\left\|\pi_{C}^{f_{2}}\left(J z_{n}-v_{n}\right)\right\| \leq r_{5} \quad$ and $\left\|\pi_{C}^{f_{3}}\left(J x_{n}-w_{n}\right)\right\| \leq r_{6}$. We choose a number $r=\max \left\{r_{1}, r_{2}, r_{3}, r_{4}, r_{5}, r_{6}\right\}$ such that $\left\{x_{n}\right\},\left\{y_{n}\right\},\left\{z_{n}\right\},\left\{\pi_{C}^{f_{1}}\left(J y_{n}-u_{n}\right)\right\},\left\{\pi_{C}^{f_{2}}\left(J z_{n}-v_{n}\right)\right\},\left\{\pi_{C}^{f_{3}}\left(J x_{n}-w_{n}\right)\right\} \subset B_{r}$, by Lemma 2.1, for $q$ $=2$ there exists a continuous, strictly increasing, and convex function $g:[0, \infty) \rightarrow[0$, $\infty)$ with $g(0)=0$ such that for $\alpha_{n}, \beta_{n}, \gamma_{n} \in[0,1]$, we have

$$
\begin{aligned}
\left\|z_{n}\right\|^{2} & =\left\|\left(1-\gamma_{n}\right) x_{n}+\gamma_{n} \pi_{C}^{f_{3}}\left(J x_{n}-w_{n}\right)\right\|^{2} \\
& \leq\left(1-\gamma_{n}\right)\left\|x_{n}\right\|^{2}+\gamma_{n}\left\|\pi_{C}^{f_{3}}\left(J x_{n}-w_{n}\right)\right\|^{2}-\gamma_{n}\left(1-\gamma_{n}\right) g\left(\left\|x_{n}-\pi_{C}^{f_{3}}\left(J x_{n}-w_{n}\right)\right\|\right) \\
& \leq\left\|x_{n}\right\|^{2}-\gamma_{n}\left(1-\gamma_{n}\right) g\left(\left\|x_{n}-\pi_{C}^{f_{3}}\left(J x_{n}-w_{n}\right)\right\|\right)
\end{aligned}
$$

and

$$
\begin{aligned}
\left\|y_{n}\right\|^{2}= & \left\|\left(1-\beta_{n}\right) x_{n}+\beta_{n} \pi_{C}^{f_{2}}\left(J z_{n}-v_{n}\right)\right\|^{2} \\
\leq & \left(1-\beta_{n}\right)\left\|x_{n}\right\|^{2}+\beta_{n}\left\|\pi_{C}^{f_{2}}\left(J z_{n}-v_{n}\right)\right\|^{2}-\beta_{n}\left(1-\beta_{n}\right) g\left(\left\|x_{n}-\pi_{C}^{f_{2}}\left(J z_{n}-v_{n}\right)\right\|\right) \\
\leq & \left(1-\beta_{n}\right)\left\|x_{n}\right\|^{2}+\beta_{n}\left\|z_{n}\right\|^{2}-\beta_{n}\left(1-\beta_{n}\right) g\left(\left\|x_{n}-\pi_{C}^{f_{2}}\left(J z_{n}-v_{n}\right)\right\|\right) \\
\leq & \left(1-\beta_{n}\right)\left\|x_{n}\right\|^{2}+\beta_{n}\left(\left\|x_{n}\right\|^{2}-\gamma_{n}\left(1-\gamma_{n}\right) g\left(\left\|x_{n}-\pi_{C}^{f_{3}}\left(J x_{n}-w_{n}\right)\right\|\right)\right) \\
& -\beta_{n}\left(1-\beta_{n}\right) g\left(\left\|x_{n}-\pi_{C}^{f_{2}}\left(J z_{n}-v_{n}\right)\right\|\right) \\
\leq & \left\|x_{n}\right\|^{2}-\beta_{n} \gamma_{n}\left(1-\gamma_{n}\right) g\left(\left\|x_{n}-\pi_{C}^{f_{3}}\left(J x_{n}-w_{n}\right)\right\|\right) \\
& -\beta_{n}\left(1-\beta_{n}\right) g\left(\left\|x_{n}-\pi_{C}^{f_{2}}\left(J z_{n}-v_{n}\right)\right\|\right) .
\end{aligned}
$$

We compute

$$
\begin{aligned}
\left\|x_{n+1}\right\|^{2}= & \left\|\left(1-\alpha_{n}\right) x_{n}+\alpha_{n} \pi_{C}^{f_{1}}\left(J y_{n}-u_{n}\right)\right\|^{2} \\
\leq & \left(1-\alpha_{n}\right)\left\|x_{n}\right\|^{2}+\alpha_{n}\left\|\pi_{C}^{f_{1}}\left(J y_{n}-u_{n}\right)\right\|^{2}-\alpha_{n}\left(1-\alpha_{n}\right) g\left(\left\|x_{n}-\pi_{C}^{f_{1}}\left(J y_{n}-u_{n}\right)\right\|\right) \\
\leq & \left(1-\alpha_{n}\right)\left\|x_{n}\right\|^{2}+\alpha_{n}\left\|y_{n}\right\|^{2}-\alpha_{n}\left(1-\alpha_{n}\right) g\left(\left\|x_{n}-\pi_{C}^{f_{1}}\left(J y_{n}-u_{n}\right)\right\|\right) \\
\leq & \left(1-\alpha_{n}\right)\left\|x_{n}\right\|^{2}+\alpha_{n}\left(\left\|x_{n}\right\|^{2}-\beta_{n} \gamma_{n}\left(1-\gamma_{n}\right) g\left(\left\|x_{n}-\pi_{C}^{f_{3}}\left(J x_{n}-w_{n}\right)\right\|\right)\right. \\
& -\beta_{n}\left(1-\beta_{n}\right) g\left(\| x_{n}-\pi_{C}^{f_{2}}\left(J z_{n}-v_{n}\right)\right)-\alpha_{n}\left(1-\alpha_{n}\right) g\left(\left\|x_{n}-\pi_{C}^{f_{1}}\left(J y_{n}-u_{n}\right)\right\|\right) \\
\leq & \left\|x_{n}\right\|^{2}-\alpha_{n} \beta_{n} \gamma_{n}\left(1-\gamma_{n}\right) g\left(\left\|x_{n}-\pi_{C}^{f_{3}}\left(J x_{n}-w_{n}\right)\right\|\right) \\
& -\alpha_{n} \beta_{n}\left(1-\beta_{n}\right) g\left(\left\|x_{n}-\pi_{C}^{f_{2}}\left(J z_{n}-v_{n}\right)\right\|\right)-\alpha_{n}\left(1-\alpha_{n}\right) g\left(\left\|x_{n}-\pi_{C}^{f_{1}}\left(J y_{n}-u_{n}\right)\right\|\right) .
\end{aligned}
$$

From above, we obtain that

$$
\begin{aligned}
\alpha_{n}\left(1-\alpha_{n}\right) g\left(\left\|x_{n}-\pi_{C}^{f_{1}}\left(J y_{n}-u_{n}\right)\right\|\right) & \leq\left\|x_{n}\right\|^{2}-\left\|x_{n+1}\right\|^{2} \\
\alpha_{n} \beta_{n}\left(1-\beta_{n}\right) g\left(\left\|x_{n}-\pi_{C}^{f_{2}}\left(J z_{n}-v_{n}\right)\right\|\right) & \leq\left\|x_{n}\right\|^{2}-\left\|x_{n+1}\right\|^{2} \\
\alpha_{n} \beta_{n} \gamma_{n}\left(1-\gamma_{n}\right) g\left(\left\|x_{n}-\pi_{C}^{f_{3}}\left(J x_{n}-w_{n}\right)\right\|\right) & \leq\left\|x_{n}\right\|^{2}-\left\|x_{n+1}\right\|^{2}{ }^{2}
\end{aligned}
$$

By the condition (iv), we have

$$
\begin{array}{r}
a(1-b) g\left(\left\|x_{n}-\pi_{C}^{f_{1}}\left(J y_{n}-u_{n}\right)\right\|\right) \leq\left\|x_{n}\right\|^{2}-\left\|x_{n+1}\right\|^{2} \\
a c(1-d) g\left(\left\|x n-\pi_{C}^{f_{2}}\left(J z_{n}-v_{n}\right)\right\|\right) \leq\left\|x_{n}\right\|^{2}-\left\|x_{n+1}\right\|^{2} \\
\operatorname{ace}(1-h) g\left(\left\|x_{n}-\pi_{C}^{f_{3}}\left(J x_{n}-w_{n}\right)\right\|\right) \leq\left\|x_{n}\right\|^{2}-\left\|x_{n+1}\right\|^{2}
\end{array}
$$


Taking the sum for $n=0,1,2, \ldots, m$ in the above inequality, we get

$$
a(1-b) \sum_{n=0}^{m} g\left(\left\|x_{n}-\pi_{C}^{f_{1}}\left(J y_{n}-u_{n}\right)\right\|\right) \leq\left\|x_{0}\right\|^{2}-\left\|x_{m+1}\right\|^{2}
$$

and

$$
\sum_{n=0}^{\infty} g\left(\left\|x_{n}-\pi_{C}^{f_{1}}\left(J y_{n}-u_{n}\right)\right\|\right)<\infty
$$

It is easy to know that

$$
\lim _{n \rightarrow \infty} g\left(\| x_{n}-\pi_{C}^{f_{1}}\left(J y_{n}-u_{n}\right)||\right)=0 .
$$

Hence, there exist subsequences $\left\{x_{n_{i}}\right\} \subset\left\{x_{n}\right\}$ and $\left\{y_{n_{i}}\right\} \subset\left\{y_{n}\right\}$ such that $u_{n_{i}} \in T_{1 y n_{i}}$ and

$$
\lim _{i \rightarrow \infty}\left\|x_{n_{i}}-\pi_{C}^{f_{1}}\left(J y_{n_{i}}-u_{n_{i}}\right)\right\|=0 .
$$

By property of functional $g$, we have

$$
\lim _{i \rightarrow \infty}\left\|x_{n_{i}}-\pi_{C}^{f_{1}}\left(J y_{n_{i}}-u_{n_{i}}\right)\right\|=0 .
$$

Similarly, we can proof that

$$
\begin{aligned}
& \lim _{i \rightarrow \infty}\left\|x_{n_{i}}-\pi_{C}^{f_{2}}\left(J z_{n_{i}}-v_{n_{i}}\right)\right\|=0, \\
& \lim _{i \rightarrow \infty}\left\|x_{n_{i}}-\pi_{C}^{f_{3}}\left(J x_{n_{i}}-w_{n_{i}}\right)\right\|=0 .
\end{aligned}
$$

Since $\left\{y_{n}\right\}$ is bounded sequence and $\left(J-T_{1}\right)$ is compact on $C$, without loss of generality there exist convergence subsequences say $\left\{J y_{n_{i}}-u_{n_{i}}\right\}$ such that

$$
\left\{J y_{n_{i}}-u_{n_{i}}\right\} \rightarrow u_{0} \in E^{*} \text { as } i \rightarrow \infty \text {. }
$$

By the continuity of the $\pi_{C}^{f_{1}}$, we have

$$
\lim _{i \rightarrow \infty} \pi_{C}^{f_{1}}\left(J y_{n_{i}}-u_{n_{i}}\right)=\pi_{C}^{f_{1}}\left(u_{0}\right) .
$$

Again since $\left\{x_{n}\right\},\left\{z_{n}\right\}$ are bounded and $\left(J-T_{2}\right),\left(J-T_{3}\right)$ are compact on $C$, without loss of generality there exist a convergence subsequence say $\left\{J x_{n_{i}}-w_{n_{i}}\right\}$ and $\left\{J z_{n_{i}}-v_{n_{i}}\right\}$ such that

$$
\left\{J x_{n_{i}}-w_{n_{i}}\right\} \rightarrow w_{0} \in E^{*} \text { as } i \rightarrow \infty,
$$

and

$$
\left\{J z_{n_{i}}-v_{n_{i}}\right\} \rightarrow v_{0} \in E^{*} \text { as } i \rightarrow \infty .
$$

By the continuity of $\pi_{C}^{f_{2}}$ and $\pi_{C}^{f_{3}}$, we have

$$
\lim _{i \rightarrow \infty} \pi_{C}^{f_{2}}\left(J z_{n_{i}}-v_{n_{i}}\right)=\pi_{C}^{f_{2}}\left(v_{0}\right)
$$

and

$$
\lim _{i \rightarrow \infty} \pi_{C}^{f_{3}}\left(J x_{n_{i}}-w_{n_{i}}\right)=\pi_{C}^{f_{3}}\left(w_{0}\right) .
$$


Let $\pi_{C}^{f_{1}}\left(u_{0}\right)=x^{*}, \pi_{C}^{f_{2}}\left(v_{0}\right)=y^{*}$, and $\pi_{C}^{f_{3}}\left(w_{0}\right)=z^{*}$.

By using the triangle inequality, we have

$$
\left\|x_{n_{i}}-x^{*}\right\| \leq\left\|x_{n_{i}}-\pi_{C}^{f_{1}}\left(J y_{n_{i}}-u_{n_{i}}\right)\right\|+\left\|\pi_{C}^{f_{1}}\left(J y_{n_{i}}-u_{n_{i}}\right)-x^{*}\right\| .
$$

From (4.6) and (4.9), we also have

$$
\lim _{i \rightarrow \infty} x_{n_{i}}=x^{*}
$$

From Algorithm 3.2 and (4.7), we obtain

$$
\left\|y_{n_{i}}-x_{n_{i}}\right\|=\beta_{n_{i}}\left\|\pi_{C}^{f_{2}}\left(J z_{n_{i}}-v_{n_{i}}\right)-x_{n_{i}}\right\| \rightarrow 0, \text { as } n \rightarrow \infty .
$$

Since above equations (4.7) and (4.12), we have

$$
\left\|y_{n_{i}}-\pi_{C}^{f_{2}}\left(J z_{n_{i}}-v_{n_{i}}\right)\right\| \leq\left\|y_{n_{i}}-x_{n_{i}}\right\|+\left\|x_{n_{i}}-\pi_{C}^{f_{2}}\left(J z_{n_{i}}-v_{n_{i}}\right)\right\| \rightarrow 0, \text { as } i \rightarrow \infty .
$$

So,

$$
\left\|y_{n_{i}}-y^{*}\right\| \leq\left\|y_{n_{i}}-\pi_{C}^{f_{2}}\left(J z_{n_{i}}-v_{n_{i}}\right)\right\|+\left\|\pi_{C}^{f_{2}}\left(J z_{n_{i}}-v_{n_{i}}\right)-y^{*}\right\| \rightarrow 0 \text {, as } i \rightarrow \infty .
$$

It follows that

$$
\lim _{i \rightarrow \infty} y_{n_{i}}=y^{*} .
$$

In the same way, we apply Algorithm 3.2, equations (4.7) and (4.13), we also have

$$
\lim _{i \rightarrow \infty} z_{n_{i}}=z^{*} .
$$

We can show that

$$
\lim _{i \rightarrow \infty} u_{n_{i}}=\lim _{i \rightarrow \infty}\left(J y_{n_{i}}-\left(J y_{n_{i}}-u_{n_{i}}\right)\right)=J y^{*}-u_{0} .
$$

Similarly, we have $\lim _{i \rightarrow \infty} v_{n_{i}}=J z^{*}-v_{0}$ and $\lim _{i \rightarrow \infty} w_{n_{i}}=J x^{*}-w_{0}$. Let $u^{*}=J y^{*}-u_{0}$, $v^{*}=J z^{*}-v_{0}$, and $w^{*}=J x^{*}-w_{0}$. Since $T_{1}, T_{2}$, and $T_{3}$ are upper semi-continuous with closed values, $T_{1}, T_{2}$, and $T_{3}$ are closed, and then $u^{*}=T_{1} y^{*}, v^{*}=T_{2} z^{*}$, and $w^{*}=T_{3} x^{*}$. It follows from Algorithm 3.2 and the continuity of the operators $\pi_{C}^{f_{1}}, \pi_{C}^{f_{2}}$, and $\pi_{C}^{f_{3}}$ that

$$
\begin{aligned}
& x^{*}=\pi_{C}^{f_{1}}\left(J y^{*}-u^{*}\right), \\
& y^{*}=\pi_{C}^{f_{2}}\left(J z^{*}-v^{*}\right), \\
& z^{*}=\pi_{C}^{f_{3}}\left(J x^{*}-w^{*}\right) .
\end{aligned}
$$

This complete of the proof.

Theorem 4.2. Let $C$ be a nonempty closed convex subset of a uniformly convex and uniformly smooth Banach space $B$ with dual space $B *$ and $0 \in C$. If the upper semicontinuous set-valued mappings $T_{1}, T_{2}, T_{3}: C \rightarrow 2^{B^{*}}$ with closed values, and the proper convex lower semi-continuous mapping $f_{1}, f_{2}, f_{3}: C \rightarrow \mathbb{R} \cup\{+\infty\}$ satisfy the following conditions:

(i) $f_{k}(x) \geq 0$ for all $x \in C$ and $f_{k}(0)=0$ for $k=1,2,3$;

(ii) for any $x \in C$ and any $z_{k} \in T_{k}(x),\left\langle z_{k}, J^{*}\left(J x-z_{k}\right)\right\rangle \geq 0$ for $k=1,2,3$;

(iii) the set-valued mappings $J-T_{k}$ are compact for $k=1,2,3$; 
(iv) $0<a \leq \alpha_{n} \leq b<1, \exists a, b \in(0,1)$.

Then problem ( $\star$ ) has a solution $\left(x^{*}, y^{*}, z^{*}\right)$ and the sequences $\left\{x_{n}\right\}$, $\left\{y_{n}\right\}$, and $\left\{z_{n}\right\}$ defined by Algorithm 3.3 have convergent subsequences $\left\{x_{n_{i}}\right\},\left\{y_{n_{i}}\right\}$, and $\left\{z_{n_{i}}\right\}$ such that $x_{n_{i}} \rightarrow x^{*}, y_{n_{i}} \rightarrow y^{*}$, and $z_{n_{i}} \rightarrow z^{*}$ as $i \rightarrow \infty$, respectively.

Proof. In this instance, (4.2) and (4.3) become

$$
\left\|z_{n}\right\| \leq\left\|\pi_{C}^{f_{3}}\left(J x_{n}-w_{n}\right)\right\| \leq\left\|x_{n}\right\|
$$

and

$$
\left\|y_{n}\right\| \leq\left\|\pi_{C}^{f_{2}}\left(J z_{n}-v_{n}\right)\right\| \leq\left\|z_{n}\right\| \leq\left\|x_{n}\right\| .
$$

Hence, the sequences $\left\{x_{n}\right\},\left\{y_{n}\right\},\left\{z_{n}\right\},\left\{\pi_{C}^{f_{1}}\left(J y_{n}-u_{n}\right)\right\},\left\{\pi_{C}^{f_{2}}\left(J z_{n}-v_{n}\right)\right\}$, and $\left\{\pi_{C}^{f_{3}}\left(J x_{n}-w_{n}\right)\right\}$ are bounded. Take a positive number $r_{1}, r_{2}, r_{3}, r_{4}, r_{5}, r_{6}$ such that $\left\|x_{n}\right\| \leq r_{1},\left\|y_{n}\right\| \leq r_{2},\left\|z_{n}\right\| \leq r_{3},\left\|\pi_{C}^{f_{1}}\left(J y_{n}-u_{n}\right)\right\| \leq r_{4},\left\|\pi_{C}^{f_{2}}\left(J z_{n}-v_{n}\right)\right\| \leq r_{5}, \quad$ and $\left\|\pi_{C}^{f_{3}}\left(J x_{n}-w_{n}\right)\right\| \leq r_{6}$. We choose a number $r=\max \left\{r_{1}, r_{2}, r_{3}, r_{4}, r_{5}, r_{6}\right\}$ such that $\left\{x_{n}\right\},\left\{y_{n}\right\},\left\{z_{n}\right\},\left\{\pi_{C}^{f_{1}}\left(J y_{n}-u_{n}\right)\right\},\left\{\pi_{C}^{f_{2}}\left(J z_{n}-v_{n}\right)\right\},\left\{\pi_{C}^{f_{3}}\left(J x_{n}-w_{n}\right)\right\}$, by Lemma 2.1, for $q=2$ there exists a continuous, strictly increasing, and convex function $g:[0, \infty) \rightarrow[0, \infty)$ with $g(0)=0$ such that for $\alpha_{n} \in[0,1]$, we have

$$
\begin{aligned}
\left\|x_{n+1}\right\|^{2} & =\left\|\left(1-\alpha_{n}\right) x_{n}+\alpha_{n} \pi_{C}^{f_{1}}\left(J y_{n}-u_{n}\right)\right\|^{2} \\
& \leq\left(1-\alpha_{n}\right)\left\|x_{n}\right\|^{2}+\alpha_{n}\left\|\pi_{C}^{f_{1}}\left(J y_{n}-u_{n}\right)\right\|^{2}-\alpha_{n}\left(1-\alpha_{n}\right) g\left(\left\|x_{n}-\pi_{C}^{f_{1}}\left(J y_{n}-u_{n}\right)\right\|\right) \\
& \leq\left(1-\alpha_{n}\right)\left\|x_{n}\right\|^{2}+\alpha_{n}\left\|y_{n}\right\|^{2}-\alpha_{n}\left(1-\alpha_{n}\right) g\left(\left\|x_{n}-\pi_{C}^{f_{1}}\left(J y_{n}-u_{n}\right)\right\|\right) \\
& \leq\left(1-\alpha_{n}\right)\left\|x_{n}\right\|^{2}+\alpha_{n}\left\|x_{n}\right\|^{2}-\alpha_{n}\left(1-\alpha_{n}\right) g\left(\left\|x_{n}-\pi_{C}^{f_{1}}\left(J y_{n}-u_{n}\right)\right\|\right) \\
& \leq\left\|x_{n}\right\|^{2}-\alpha_{n}\left(1-\alpha_{n}\right) g\left(\mid x_{n}-\pi_{C}^{f_{1}}\left(J y_{n}-u_{n}\right) \|\right) .
\end{aligned}
$$

Similarly proof of Theorem 4.1, we obtain that

$$
\lim _{i \rightarrow \infty}\left\|x_{n_{i}}-\pi_{C}^{f_{1}}\left(J y_{n_{i}}-u_{n_{i}}\right)\right\|=0 .
$$

Since $\left\{y_{n}\right\},\left\{z_{n}\right\}$, and $\left\{x_{n}\right\}$ are bounded sequences, $\left(J-T_{1}\right),\left(J-T_{2}\right)$, and $\left(J-T_{3}\right)$ are compact on $C$ and by the continuity of the $\pi_{C}^{f_{1}}, \pi_{C}^{f_{2}}$, and $\pi_{C}^{f_{3}}$, we have

$$
\begin{aligned}
& \lim _{i \rightarrow \infty} \pi_{c}^{f_{1}}\left(J y_{n_{i}}-u_{n_{i}}\right)=\pi_{C}^{f_{1}}\left(u_{0}\right)=x^{*} \\
& \lim _{i \rightarrow \infty} \pi_{c}^{f_{2}}\left(J z_{n_{i}}-v_{n_{i}}\right)=\pi_{C}^{f_{2}}\left(v_{0}\right)=y^{*} \\
& \lim _{i \rightarrow \infty} \pi_{c}^{f_{3}}\left(J x_{n_{i}}-w_{n_{i}}\right)=\pi_{C}^{f_{3}}\left(w_{0}\right)=z^{*} .
\end{aligned}
$$

Hence, we obtain that $\lim _{i \rightarrow \infty} x_{n_{i}}=x^{*}$.

By Algorithm 3.3, we get

$$
\left\|y_{n_{i}}-\gamma^{*}\right\| \leq\left\|\pi_{C}^{f_{2}}\left(J z_{n_{i}}-v_{n_{i}}\right)-\gamma^{*}\right\|
$$

and

$$
\left\|z_{n_{i}}-z^{*}\right\| \leq\left\|\pi_{C}^{f_{3}}\left(J x_{n_{i}}-w_{n_{i}}\right)-z^{*}\right\| .
$$


It follows from above, we obtain that $\lim _{i \rightarrow \infty} y_{n_{i}}=y^{*}$ and $\lim _{i \rightarrow \infty} z_{n_{i}}=z^{*}$. Similarly to the proof of Theorem 4.1, we can obtain this Theorem.

If $f_{1}(x)=f_{2}(x)=f_{3}(x)=0, \forall x \in C$, then the following theorem can be obtained from Theorem 4.1 directly.

Corollary 4.3. Let $C$ be a nonempty, closed convex subset of a uniformly convex and uniformly smooth Banach space $B$ with dual space $B^{*}$ and $0 \in C$. If the upper semicontinuous set-valued mappings $T_{1}, T_{2}, T_{3}: C \rightarrow 2^{B^{*}}$ with closed values satisfy the following conditions:

(i) for any $x \in C$ and any $z_{k} \in T_{k}(x),\left\langle z_{k}, J^{*}\left(J x-z_{k}\right)\right\rangle \geq 0$ for $k=1,2,3$;

(ii) the set-valued mappings $J-T_{k}$ are compact for $k=1,2,3$;

(iii) $0<a \leq \alpha_{n} \leq b<1,0<c \leq \beta_{n} \leq d<1$ and $0<e \leq \gamma_{n} \leq h<1, \exists a, b, c, d, e, h \in$ $(0,1)$.

Then problem (1.1) has a solution $\left(x^{*}, y^{*}, z^{*}\right)$ and the sequences $\left\{x_{n}\right\},\left\{y_{n}\right\}$, and $\left\{z_{n}\right\}$ defined by Algorithm 3.4 have convergent subsequences $\left\{x_{n_{i}}\right\},\left\{y_{n_{i}}\right\}$, and $\left\{z_{n_{i}}\right\}$ such that $x_{n_{i}} \rightarrow x^{*}, y_{n_{i}} \rightarrow y^{*}$, and $z_{n_{i}} \rightarrow z^{*}$ as $i \rightarrow \infty$, respectively.

If $x_{n}=z_{n}, f_{3}(x)=0$, and $T_{3}(y)=0, \forall x, y \in C$, then the following theorem can be obtained from Theorem 4.1 directly.

Corollary 4.4. Let $C$ be a nonempty closed convex subset of a uniformly convex and uniformly smooth Banach space $B$ with dual space $B^{*}$ and $0 \in C$. If the upper semicontinuous set-valued mappings $T_{1}, T_{2}: C \rightarrow 2^{B^{*}}$ with closed values and the proper convex lower semi-continuous mapping $f_{1}, f_{2}: C \rightarrow \mathbb{R} \cup\{+\infty\}$ satisfy the following conditions:

(i) $f_{k}(x) \geq 0$ for all $x \in C$ and $f_{k}(0)=0$ for $k=1,2$;

(ii) for any $x \in C$ and any $z_{k} \in T_{k}(x),\left\langle z_{k}, J^{*}\left(J x-z_{k}\right)\right\rangle \geq 0$ for $k=1,2$;

(iii) the set-valued mappings $J$ - $T_{k}$ are compact for $k=1,2$;

(iv) $0<a \leq \alpha_{n} \leq b<1$ and $0<c \leq \beta_{n} \leq d<1, \exists a, b, c, d \in(0,1)$.

Then problem (1.2) has a solution $\left(x^{*}, y^{*}\right)$ and the sequences $\left\{x_{n}\right\}$ and $\left\{y_{n}\right\}$ defined by Algorithm 3.5 have convergent subsequences $\left\{x_{n_{i}}\right\}$ and $\left\{y_{n_{i}}\right\}$ such that $x_{n_{i}} \rightarrow x^{*}$ and $y_{n_{i}} \rightarrow \gamma^{*}$ as $i \rightarrow \infty$, respectively.

If $x_{n}=y_{n}=z_{n}, T=T_{1}=T_{2}=T_{3}$, and $f_{2}(x)=f_{3}(x)=0, \forall x \in C$, then the following theorem can be obtained from Theorem 4.1 directly.

Corollary 4.5. Let $C$ be a nonempty closed convex subset of a uniformly convex and uniformly smooth Banach space B with dual space $B^{*}$ and $0 \in C$. If the upper semicontinuous set-valued mappings $T: C \rightarrow 2^{B^{*}}$ with closed values and the proper convex lower semi-continuous mapping $f_{1}: C \rightarrow \mathbb{R} \cup\{+\infty\}$ satisfy the following conditions:

(i) $f_{1}(x) \geq 0$ for all $x \in C$ and $f_{1}(0)=0$;

(ii) for any $x \in C$ and any $z \in T(x),\left\langle z, J^{*}(J x-z)\right\rangle \geq 0$;

(iii) the set-valued mappings $J$ - $T$ is compact;

(iv) $0<a \leq \alpha_{n} \leq b<1, \exists a, b \in(0,1)$.

Then problem (1.3) has a solution $x^{*}$ and the sequence $\left\{x_{n}\right\}$ defined by Algorithm 3.6 has a convergent subsequence $\left\{x_{n_{i}}\right\}$ such that $x_{n_{i}} \rightarrow x^{*}$ as $i \rightarrow \infty$. 
Remark 4.6. Theorems 4.1, 4.2, and Corollary 4.3 generalize and improve the main result in [1].

Our results generalize and extend the main result of [8] from a Hilbert space to a Banach space.

\section{Acknowledgements}

This research was partially supported by the "Centre of Excellence in Mathematics", the Commission on High Education, Ministry of Education, Thailand (under the project No. RG-1-53-03-2). Moreover, Mr. Nawitcha Onjai-uea was supported by the "Centre of Excellence in Mathematics", the Commission on High Education for the Ph.D. Program at KMUTT.

\section{Author details}

'Department of Mathematics, Faculty of Science, King Mongkut's University of Technology Thonburi (KMUTT), Bangkok 10140, Thailand ${ }^{2}$ Centre of Excellence in Mathematics, CHE, Sriayudthaya Rd., Bangkok 10140, Thailand

\section{Authors' contributions}

All authors contributed equally and significantly in this research work. All authors read and approved the final manuscript.

\section{Competing interests}

The authors declare that they have no competing interests.

Received: 4 August 2011 Accepted: 16 January 2012 Published: 16 January 2012

\section{References}

1. Zhang, Q-b, Deng, R: Projection algorithms for the system of generalized mixed variational inequalities in Banach spaces. Appl Math Comput. (2011)

2. Fan, J, Liu, X, Li, J: Iterative schemes for approximating solutions of generalized variational inequalities in Banach spaces. Nonlinear Anal. 70, 3997-4007 (2009). doi:10.1016/j.na.2008.08.008

3. Stampacchia, G: Formes bilinearies coercivities sur les ensembles convexes. CR Acad Sci Paris. 258, 4413-4416 (1964)

4. Noor, MA: New approximation schemes for general variational inequalities. J Math Anal Appl. 251, 217-229 (2000). doi:10.1006/jmaa.2000.7042

5. Noor, MA: New extragradient-type methods for general variational inequalities. J Math Anal Appl. 277, 379-395 (2003). doi:10.1016/S0022-247X(03)00023-4

6. Noor, MA: Projection-proximal methods for general variational inequalities. J Math Anal Appl. 316, 53-62 (2006)

7. Noor, MA: Some developments in general variational inequalities. Appl Math Comput. 152, 199-277 (2004). doi:10.1016/ S0096-3003(03)00558-7

8. Petrot, N: A resolvent operator technique for approximate solving of generalized system mixed variational inequality and fixed point problems. Appl Math Lett. 23(4), 440-445 (2010). doi:10.1016/j.aml.2009.12.001

9. Verma, RU: A class of projection-contraction methods applied to monotone variational inequality. Appl Math Lett. 13, 55-62 (2000)

10. Verma, RU: General convergence analysis for two-step projection methods and application to variational problems. Appl Math Lett. 18, 1286-1292 (2005). doi:10.1016/j.aml.2005.02.026

11. Alber, Ya: Metric and generalized projection operators in Banach spaces: Properties and applications. In: Kartsatos, A (eds.) Theory and Applications of Nonlinear Operators of Accretive and Monotone Type. pp. 15-50. Dekker, New York (1996)

12. Li, JL: The generalized projection operator on reflexive Banach spaces and its applications. J Math Anal Appl. 306 55-71 (2005). doi:10.1016/j.jmaa.2004.11.007

13. $\mathrm{Wu}, \mathrm{KQ}$, Huang, NJ: Properties of the generalized f-projection operator and its applications in Banach spaces. Comput Math Appl. 54, 399-406 (2007). doi:10.1016/j.camwa.2007.01.029

14. Xu, HK: Inequalities in Banch spaces with applications. Nonlinear Anal. 16, 1127-1138 (1991). doi:10.1016/0362-546X(91) 90200-K

15. Chang, SS: On Chidumes open questions and approximate solutions of multivalued strongly accretive mapping in Banach spaces. J Math Anal Appl. 216, 94-111 (1997). doi:10.1006/jmaa.1997.5661

16. $\mathrm{Wu}, \mathrm{KQ}$, Huang, NJ: The generalised f-projection operator with an application. Bull Aust Math Soc. 73, 307-317 (2006). doi:10.1017/S0004972700038892

doi:10.1186/1029-242X-2012-9

Cite this article as: Onjai-uea and Kumam: Existence and convergence theorems for the new system of generalized mixed variational inequalities in Banach spaces. Journal of Inequalities and Applications 2012 2012:9. 